\section{Der Stoffhaushalt nationaler Volkswirtschaften und Aspekte eines nachhaltigen Ressour- cenmanagements}

\author{
von Stefan Bringezu, Stephan Moll und \\ Helmut Schütz, Wuppertal Institut für Klima, \\ Umwelt, Energie
}

Der Stoffhaushalt einer Gesellschaft bestimmt den Stoffwechsel mit der Umwelt, d. $h$. sämtliche Stoffentnahmen und -abgaben aus bzw. an die Natur. Die möglichen Wirkungen dieser Stoffströme auf die Umwelt lassen sich mit Hilfe von Indikatoren beschreiben, wobei wir zwischen wirkungsund mengenumsatzbasierten Indikatoren unterscheiden. Wir stellen die Methode wirtschaftsraumbezogener Stoffstromanalysen vor und davon abgeleitete Input-, Output-, Verbrauchs-, Bilanz- und EffizienzIndikatoren. Der Globale Materialaufwand der Europäischen Union (EU-15) wird anhand empirischer Befunde erläutert. Die Ergebnisse werden vor dem Hintergrund aktueller politischer Zielvorgaben auf europäischer Ebene diskutiert.

\section{„Was ist nationaler Stoffhaushalt?“}

Konzept des "Gesellschaftlichen Stoffwechsels", Systemansatz

Das systemanalytische Konzept des „Gesellschaftlichen Stoffwechsels "“ (Ayres 1989, Ayres, Simonis 1994, Baccini, Brunner 1991, Fischer-Kowalski et al. 1997) basiert auf den thermodynamischen Gesetzen über die Erhaltung von Materie und Energie. Das Konzept geht von einem einfachen Umwelt-GesellschaftModell aus (Abb. 1), wobei die Gesellschaft in die umgebende Umwelt eingebettet und über Stoff ${ }^{2}$ - und Energieströme mit ihr verbunden ist (Georgescu-Roegen 1971, Daly 1991 und 1997, Bringezu 1993, Strassert 1993). „Gesellschaft"“ bezieht sich dabei zunächst auf die globale Anthroposphäre. Für die gesellschaftlichen Akteure ist jedoch eher interessant, was dieser Stoffaustausch für ihre Subsysteme bedeutet, d. h. die nationale Volkswirtschaft, eine Region oder auch kleinere gesellschaftliche Systeme wie eine Stadt oder industrielle Prozesse. Die folgenden Ausführungen beziehen sich auf nati- onale Volkswirtschaften bzw. den Wirtschaftsraum der Europäischen Union (EU-15).

Die physischen Stoffwechselbeziehungen zwischen Gesellschaft und Umwelt - der Stoffhaushalt - umfassen die Extraktion von Rohstoffen, deren Umwandlung in Produkte und Dienstleistungen, sowie die Ab(Rück-)gabe an die Umwelt in Form von Emissionen und Abfällen.

Alle diese Stoffwechselvorgänge werden durch Energie ,angetrieben“. Die Quantität, Struktur und Qualität dieses physischen Durchsatzes ist Gegenstand der Diskussion über ein „Nachhaltiges Ressourcenmanagement", d. h. wie die menschlich verursachten Stoff- und Energieströme in einer nachhaltigen Art und Weise gestaltet werden könnten.

Abb. 1: Umwelt-Gesellschaft-Modell

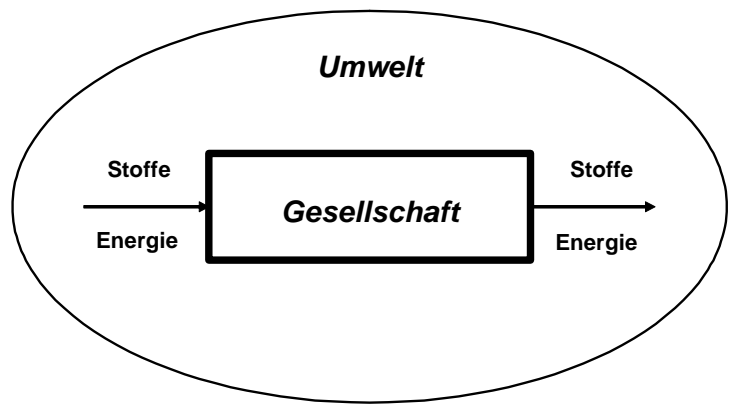

Spektrum der Auswirkungen von Stoffströmen auf die Umwelt

Viele Umweltveränderungen stehen im $\mathrm{Zu}$ sammenhang mit den vom Menschen verursachten Stoffströmen. Die Auswirkungen können vielfältig sein und in ihrer Konsequenz kurzfristig oder langfristig, direkt oder indirekt, lokal bis global, vorhersagbar oder unbestimmt sein. Stoffliche Auswirkungen umfassen (Bringezu 1997):

- (öko-)toxikologische Effekte,

- physiko-chemische Veränderungen (z. B. Versauerung etc.),

- Wirkungen auf den Nährstoffkreislauf (z. B. Eutrophierung),

- mechanische Zerstörungen (z. B. durch Baumaßnahmen, Erosion etc.),

- Wirkungen auf biotische Strukturen (z. B. Landschaftsveränderungen, Zerschneidung natürlicher Habitate etc.). 
Die Bewertung von menschlich verursachten Stoffströmen und Stoffakkumulationen kann zum einen an ihrer Qualität (substanzspezifische Effekte) oder Quantität (systemspezifische Effekte) ansetzen (Bringezu 2000). So bestimmen beispielsweise die chemischen Eigenschaften einer Substanz deren (Öko-)Toxizität und in der Folge sprechen wir von ,gefährlichen Stoffen“. Sind solche substanz-spezifischen Effekte bekannt, können sie in Form wirkungs-basierter Indikatoren gemessen und operationalisiert werden (z. B. ozone depletion potenzial, ODP).

Allerdings gibt es auch Umweltauswirkungen menschlich verursachter Stoffströme, die nicht substanz-spezifisch sind. So sind beispielsweise die Landschaftsveränderungen im Zusammenhang mit Bergbauaktivitäten eher abhängig von der Menge der abgebauten Rohstoffe als von deren chemischen Eigenschaften. Je nach dem natürlichen Inventar vor dem Abbau ist dies auch gültig für die Auswirkungen auf die Biodiversität. Ein weiteres Problem sind die Flächen, die durch den Abbau nichterneuerbarer Ressourcen für den Anbau erneuerbarer Ressourcen „verloren“ gehen. Dasselbe gilt für die Versiegelung von Flächen durch Gebäude und Infrastrukturen. Solche systembezogenen Auswirkungen von Menschen verursachter Stoffströme können mit Indikatoren erfasst werden, die das Wirkungspotenzial auf ein bestimmtes System, wie beispielsweise die in die globale Ökosphäre eingebettete nationale Volkswirtschaft, widerspiegeln. Solche Indikatoren, die auf ein systemspezifisches Wirkungspotenzial abstellen, sind eher mengenumsatzbasiert, wie beispielsweise Energieverbrauch, Materialaufwand oder Wasserverbrauch.

In ihrer Bedeutung für die Umweltpolitik ergänzen sich substanzspezifische wirkungsbasierte Indikatoren einerseits und systemspezifische mengenumsatzbezogene Indikatoren andererseits. Traditionell orientierte sich stoffbezogene Umweltpolitik zunächst weitgehend an wirkungsbasierten Indikatoren. In Deutschland beschäftigte sich erstmals gegen Mitte der 1990er Jahre eine Enquete-Kommission systematischer mit dem Stoffhaushalt der deutschen Volkswirtschaft (Enquete-Kommission 1994, 1998). Parallel dazu begann die amtliche Statistik im Rahmen der umweltökonomischen Gesamtrechnungen mit ganzheitlichen Materi- albilanzen, die im folgenden Abschnitt kurz vorgestellt werden.

\section{Methoden zur Erfassung des Stoffhaushal- tes nationaler Volkswirtschaften}

\section{Kurzer Überblick über methodische Entwick- lungen}

Methoden zur Erfassung des Stoffhaushaltes nationaler Volkswirtschaften wurden zunächst im wissenschaftlichen Bereich entwickelt und später nach und nach in den amtlich-statistischen Bereich übertragen. Dabei hat sich die englische Bezeichnung, Material Flow Accounting" oder "Material Flow Analysis“ (MFA) als Oberbegriff für verschiedenste Methoden der Stoffstromanalyse etabliert (Bringezu, Moriguchi 2002). Stoffstromanalysen (MFA) sind Bilanzierungssysteme in physischen Einheiten (gewöhnlich kg oder Tonnen pro Zeiteinheit), mit deren Hilfe der Fluss von Stoffen durch die verschiedenen Prozesse von der Rohstoffgewinnung, über Produktion und Konsumption bis hin zum Abfallmanagement quantitativ erfasst und dargestellt wird (Bringezu 2000). Grundlegende methodische Vorarbeiten wurden am Wuppertal Institut geleistet (Schmidt-Bleek et al. 1998, Bringezu, Schütz 1995) und bildeten die Basis für international vergleichende Studien (Adriaanse et al. 1998, Matthews et al. 2000). Sie gaben zudem Anstoß zur Bildung eines internationalen wissenschaftlichen Netzwerkes, das sich mit dem Gesamtspektrum stoffflussanalytischer Instrumente befasst (ConAccount Coordination of regional and national material flows accounting for sustainability; siehe http://www.conaccount.net) (Bringezu et al. 1997, 1998a, 1998b).

Das Statistische Bundesamt richtete Anfang der 1990er Jahre die „Umweltökonomischen Gesamtrechnungen" (UGR) als umweltrelevante Ergänzung $\mathrm{zu}$ den herkömmlichen Volkswirtschaftlichen Gesamtrechnungen ein (Radermacher, Stahmer 1994, 1995). Ein wesentlicher Baustein der UGR sind die „Materialund Energieflussrechnungen“, deren Ergebnisse regelmäßig veröffentlicht werden ${ }^{3}$. In der vom Statistischen Bundesamt eingerichteten „Arbeitsgemeinschaft Material- und Energieflussrechnungen" (AGME) wurden begleitend methodische Fragen im Expertenkreis erörtert (Sta- 
tistisches Bundesamt 1997, Bringezu 1995). Beim Umweltbundesamt (UBA) wurde ein Fachgebiet „Stoffflüsse“ eingerichtet.

Auf internationaler Ebene beschäftigt sich die so genannte „London-Group“ mit Methoden der Stoffflussrechnungen im Zusammenhang mit dem Vorschlag der Vereinten Nationen zu einem integrierten System umweltökonomischer Gesamtrechnungen (System of Integrated Environmental and Economic Accounting, SEEA) (United Nations 1993, 2000).

Das statistische Amt der Europäischen Gemeinschaften (Eurostat) bemüht sich insbesondere um die Harmonisierung und Standardisierung der Methoden zur Erfassung nationaler Stoffhaushalte. Beraten von einer internationalen Task Force und in Zusammenarbeit mit dem Wuppertal Institut wurde ein methodisches Handbuch zur wirtschaftsraumbezogenen Stoffflussbilanzierung und abgeleiteten Indikatoren veröffentlicht (Eurostat 2001). Die wichtigsten Bilanzierungs-Konventionen letztgenannter Publikation werden im folgenden $\mathrm{Ab}$ schnitt vorgestellt.

\section{EUROSTAT Methodenhandbuch zu Stoff- strombilanzen (economy-wide material flow accounts and derived indicators)}

Wirtschaftsraumbezogene Stoffflussbilanzen (economy-wide MFA) bieten einen Überblick über die jährlichen Materialinputs und Materialoutputs einer nationalen Volkswirtschaft in physischen Einheiten (Tonnen pro Jahr). Auf der Inputseite umfassen sie sowohl die Primärmaterialentnahmen aus der inländischen Umwelt wie auch die Importe und deren ,ökologische Rucksäcke“, d. h. die indirekten lebenszyklusweiten Primärmaterialentnahmen, die notwendig waren, um das Importgut herzustellen. Auf der Outputseite umfassen sie die Stoffabgaben an die inländische Umwelt sowie die Exporte und deren „ökologischen Rucksäcke“. Außerdem weisen sie die jährliche Nettoveränderung des Materialbestandes der Volkswirtschaft aus (zusätzliche Gebäude und Infrastrukturen), welcher sich aus der Differenz zwischen Input und Output ergibt (Abb. 2).
Abb. 2: Allgemeines Schema einer wirtschaftsraumbezogenen Stoffflussbilanz ohne Wasser und Luft

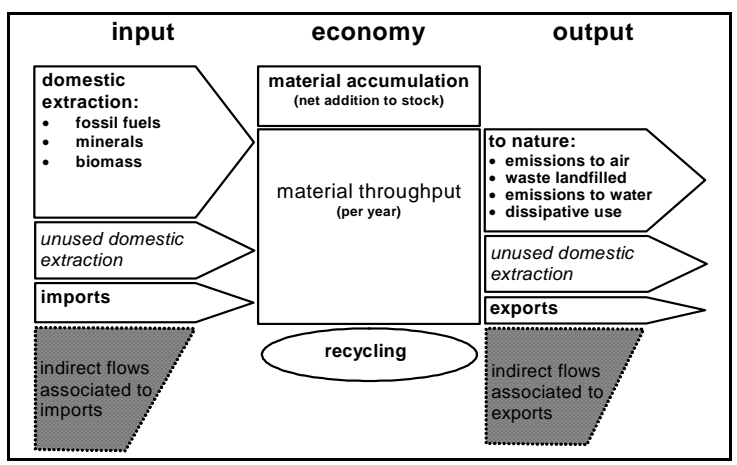

Quelle: Eurostat 2001

Diese wirtschaftsraumbezogenen Stoffflussbilanzen bilden die Grundlage für eine Reihe von aggregierten Indikatoren, die über wesentliche Ausprägungsmerkmale des gesellschaftlichen Stoffwechsels informieren. Dazu gehören Input-, Output-, Verbrauchs-, Bilanz- und Effizienz-Indikatoren (Tab. 1). Wir gehen hier nur auf die wichtigsten ein. Ausführlichere Informationen geben Bringezu und Schütz (2001c), Eurostat (2001), Bringezu und Moriguchi (2002).

\section{- Input-Indikatoren}

Direkter Material Input (DMI; engl.: Direct Material Input, DMI) - dieser Indikator misst die direkt eingesetzten Materialinputs, die zur Weiterverarbeitung in der jeweiligen Volkswirtschaft dienen und demzufolge über einen ökonomischen Wert verfügen. Es sind dies die verwerteten inländischen Ressourcenentnahmen und die Einfuhren.

Globaler Materialaufwand (GMA; engl.: Total Material Requirement, TMR) - umfasst zusätzlich zum DMI die ,ökologischen Rucksäcke" der inländischen Förderung und der Importe. Erstere umfassen die nicht verwerteten Stoffentnahmen sowie vergleichbare Stoffbewegungen im Inland (z. B. Abraum, Bodenaushub, Erosion). Letztere umfassen die indirekten Primärmaterialentnahmen der Importe, die lebenszyklusweit notwendig waren, um das Importgut herzustellen. Der GMA ist der umfassendste Input-Indikator und misst die materielle Basis einer Volkswirtschaft, d. h. alle der Umwelt im In- oder Ausland entnommenen Primärmateria- 
lien, die mit der inländischen Produktion verbunden sind. Der GMA ist damit der beste Schätzwert für die Größenordnung der potenziellen Umweltbelastungen durch die Entnahme und Nutzung natürlicher stofflicher Ressourcen.

\section{- Output-Indikatoren}

Verarbeitete Stoffabgabe (VSA; engl.: Domestic Processed Output, DPO) - sind die direkten stofflichen Outputs von inländischen Wirtschaftsaktivitäten an die inländische Umwelt. Dieser Indikator umfasst Luftemissionen, Abfälle, die stoffliche Fracht von Abwässern, sowie dissipative stoffliche Verluste wie beispielsweise verursacht durch Produktabnutzung oder landwirtschaftliche Düngung.

Inländische Stoffabgabe (ISA; engl.: Total Domestic Output, TDO) - ist die Summe aus VSA und den nicht-verwerteten inländischen Entnahmen. Dieser Indikator umfasst die Gesamtheit aller stofflichen Outputs an die inländische Umwelt.

\section{- Verbrauchsindikatoren}

Inländischer Materialverbrauch (IMV; engl.: Domestic Material Consumption, DMC) - ist definiert als DMI minus Ausfuhren. Er misst die Gesamtheit der Stoffe, die direkt in einer Volkswirtschaft verbraucht werden.

Globaler Materialverbrauch (GMV; engl.:

Total Material Consumption, TMC) - ist definiert als GMA abzüglich der Ausfuhren und ihrer „ökologischen Rucksäcke“. Er misst alle direkten und indirekten Primärmaterialentnahmen, sowohl im Inland wie im Ausland, die mit dem Konsum einer Volkswirtschaft verbunden sind.

\section{- Bilanz-Indikatoren}

Netto-Bestands-Zuwachs (NBZ, engl.: Net Additions to Stock, NAS) - ergibt sich aus der Differenz zwischen dem Materialinput und Materialoutput einer Volkswirtschaft (DMI minus DPO). Er misst somit das ,physische Wachstum" einer Volkswirtschaft, d.h. die Netto-Gewichtszunahme durch neue Gebäude, Infrastrukturen und langlebige Güter (z. B. Fahrzeuge, Maschinen etc.).

Physische Handelsbilanz (PHB, engl.: Physical Trade Balance, PTB) - entspricht der physischen Differenz zwischen Einfuhren minus Ausfuhren und misst somit die physische Handelsbilanz. Aussagekräftig wird der Wert zum einen, wenn er nach einzelnen Substanzen aufgelöst wird, und zum anderen, wenn zusätzliche Parameter einbezogen werden. So können die ökologischen Rucksäcke der Importe und Exporte einbezogen werden, um die Frage zu klären, ob ein Wirtschaftsraum über den Außenhandel mehr Primärmaterial von außerhalb aufwendet (Import) als er nach außen zur Verfügung stellt (Export).

\section{- Effizienz-Indikatoren}

Effizienzmaße lassen sich für die Gesamtwirtschaft ableiten, indem das erwünschte Ergebnis wirtschaftlicher Aktivität in Beziehung zu den verschiedenen Stoffstromparametern gesetzt wird. Wenngleich das Brutto-Inlands-Produkt (BIP) auch nicht als Wohlstandsindikator geeignet ist, so zeigt es doch den Umfang der wirtschaftlichen Aktivitäten an.

Direkte Material Produktivität - weist das Verhältnis von BIP zu DMI aus. Die Direkte Material Produktivität bringt zum Ausdruck, wie viel Wertschöpfung aus einer Tonne DMI erwirtschaftet wird. Ihre Entwicklung über die Zeit gibt Hinweise darauf, inwieweit eine Abkopplung von erzielter Wertschöpfung und direktem Materialaufwand stattfindet. 
Tab. 1: Allgemeines Bilanzierungsschema einer wirtschaftsraumbezogenen Stoffstrombilanz und abgeleitete Indikatoren

\begin{tabular}{|c|c|}
\hline INPUTS (origin) & OUTPUTS (destination) \\
\hline Domestic extraction (used) & Emissions and wastes \\
\hline Fossil fuels (coal, oil...) & Waste landfilled \\
\hline Minerals (ores, gravel...) & Emissions to air \\
\hline Biomass (timber, cereals...) & Emissions to water \\
\hline & Dissipative use of products and dissipative losses \\
\hline & Dissipative use of products \\
\hline Imports & Dissipative losses \\
\hline DMI - direct material inputs & DPO - domestic processed output to nature \\
\hline Unused domestic extraction & Disposal of unused materials domestically extracted \\
\hline ......from mining/quarrying & ......from mining/quarrying \\
\hline biomass from harvest & biomass from harvest \\
\hline soil excavation and dredging & soil excavation and dredging \\
\hline \multirow[t]{3}{*}{ TMI - total material input } & TDO - total domestic output to nature \\
\hline & Exports \\
\hline & TMO - total material output \\
\hline Indirect flows associated with imports & Net Additions to Stock \\
\hline \multirow[t]{3}{*}{ TMR - total material requirements } & Infrastructures and buildings \\
\hline & Other (machinery, durable goods, etc.) \\
\hline & Indirect flows associated with exports \\
\hline
\end{tabular}

Hinweis: ohne Luft und Wasser (soweit nicht in anderen Stoffen enthalten)

Quelle: Eurostat 2001

\section{Einige wichtige empirische Ergebnisse - Der Stoffhaushalt der EU}

Im folgenden Abschnitt wird der Stoffhaushalt der Europäischen Union charakterisiert. Die Ergebnisse beruhen weitgehend auf empirischen Studien, die das Wuppertal Institut für das Statistische Amt der Europäischen Gemeinschaften (Eurostat) und die Europäische Umweltagentur (EEA) erstellt hat (Bringezu, Schütz 2001a, 2001b, 2001c). Alle im Folgenden angeführten Angaben in Tonnen beziehen sich im jeweils angegebenen Zeitraum auf die Periode von einem Jahr.

Seit 1980 schwankt der Globale Materialaufwand (GMA) der EU Wirtschaft um 51 bis 52 Tonnen pro Einwohner (Abb. 3).
Dieser mehr oder weniger konstante Ressourcenverbrauch bedeutet eine relative $\mathrm{Ab}$ kopplung vom Wirtschaftswachstum. Entsprechend ist die Ressourcenproduktivität der EU gemessen als Verhältnis reales Bruttoinlandsprodukt (BIP) zum GMA - zwischen 1980 und 1997 kontinuierlich angestiegen. Diese Produktivitätssteigerung war jedoch zu gering, um einen absoluten Rückgang des Globalen Materialaufwandes zu bewirken. Um eine absolute Abkopplung von Ressourcenverbrauch und Wirtschaftswachstum zu ermöglichen, müssten die Wachstumsraten der Ressourcenproduktivität rascher ansteigen als die des BIP. Dies bedeutet, dass einerseits der Markt sich bereits in Richtung einer erhöhten Ressourcenproduktivität entwickelt. Andererseits hat der technologische 
Wandel in Richtung ressourceneffizienter Technologien bislang nicht zu einer absoluten Ressourcenentlastung der Umwelt geführt.

\section{Abb. 3: Abkopplung von Globalem Material- aufwand (GMA) und Wirtschafts- wachstum (BIP) in der Europäischen Union}

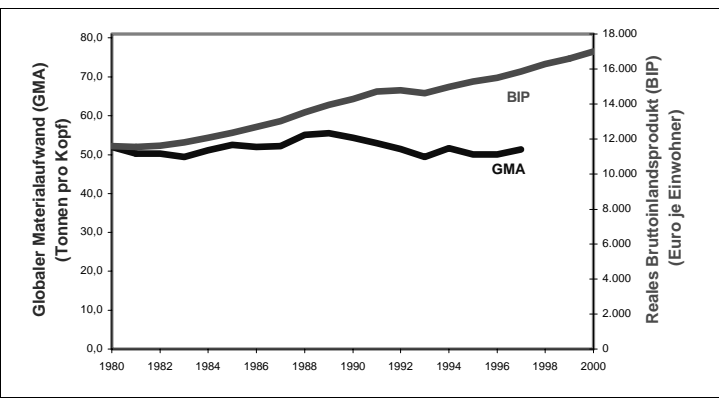

Quelle: Bringezu, Schütz 2001c

Der Globale Materialaufwand der Europäischen Union wird in seiner stofflichen Zusammensetzung von nicht-erneuerbaren Ressourcen dominiert. Der Anteil der nicht-erneuerbaren Ressourcen lag in den beiden vergangenen Jahrzehnten praktisch konstant bei rund $88 \%$ - einem sicherlich nicht-nachhaltigem Niveau. Fossile Energieträger bilden mit rund 15 Tonnen/ Kopf eine maßgebliche Komponente des GMA. Rund die Hälfte davon sind nicht-verwertete inländische Entnahmen (z. B. Abraum aus dem Bergbau). Industrielle und Baumineralien sind mit rund 12 Tonnen/Kopf eine weitere wichtige Stoffkategorie. Metalle bilden mit rund 10 Tonnen/Kopf die dritte wichtige Stoffgruppe innerhalb des GMA.

Der Anteil der inländischen und ausländischen „ökologischen Rucksäcke“ am gesamten Materialaufwand der Europäischen Union ist leicht gesunken. Dies deutet eine leicht effizientere Bereitstellung von primären Ressourcen an. Der Anteil der ausländischen Ressourcen - Einfuhren plus ihrer ,ökologischen Rucksäcke“ - ist allerdings seit den späten 1980er Jahren von rund $30 \%$ auf $39 \%$ angestiegen (Abb. 4). Dies impliziert eine Zunahme der von der EU Wirtschaft ausgehenden potenziellen Umweltbelastungen im Ausland. Dabei handelt es sich um einen eindeutigen Verlagerungseffekt, denn die innereuropäischen Ressourcenentnahmen - mit denen wiederum Ex- porte ermöglicht werden - sind im gleichen Zeitraum gesunken.

\section{Abb. 4: Stoffliche Zusammensetzung des Glo- balen Materialaufwands (GMA) der Europäischen Union}

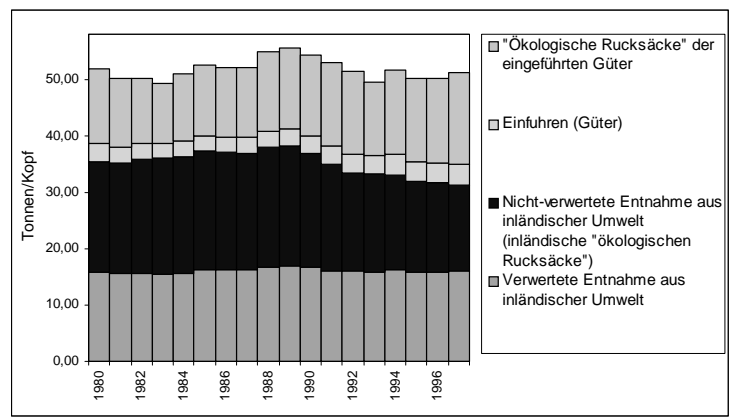

Quelle: Bringezu, Schütz 2001c

Als besorgniserregend kann das physische Wachstum der EU Wirtschaft bezeichnet werden. Der Netto-Bestands-Zuwachs NBZ (Gebäude, Infrastrukturen, langlebige Güter etc.) betrug während der 1990er Jahre jährlich rund 10 Tonnen/Kopf (Bringezu, Schütz 2001c). Diese akkumulierenden Stofflager konstituieren latente zukünftige Abfallströme. Außerdem ist dieses physische Wachstum auch eng mit der Problematik der zunehmenden Siedlungs- und Verkehrsflächenzunahme verknüpft.

\section{Aspekte eines nachhaltigen Ressourcen- managements}

\section{Politischer Hintergrund}

Das nachhaltige Management von natürlichen Ressourcen gewinnt in jüngster Zeit an umweltpolitischer Bedeutung. Die Strategie für eine nachhaltige Entwicklung der Europäischen Union formuliert als ein grundlegendes Ziel die Abkopplung von Wirtschaftswachstum und Ressourcennutzung (Kommission der Europäischen Gemeinschaften 2001b). Im aktuellen sechsten Umweltaktionsprogramm der Europäischen Union (Kommission der Europäischen Gemeinschaften 2001a) wird für den Schwerpunktbereich „Nachhaltige Nutzung natürlicher Ressourcen und Abfallwirtschaft" eine Situation angestrebt, in der die Tragfähigkeit der Umwelt durch den Verbrauch von erneuerbaren und nicht-erneuerbaren Ressourcen nicht überstiegen wird. Ein weiteres formuliertes Ziel ist auch hier 
die Abkopplung von Wirtschaftswachstum und Ressourcenverbrauch durch eine deutlich rationellere Ressourcennutzung, eine Dematerialisierung der Wirtschaft und eine effektive Abfallvermeidung. Die Europäische Kommission wird im Laufe des Jahres eine thematische Strategie zum Thema ,Nachhaltige Nutzung der Ressourcen" erarbeiten, welche die eher generellen Aussagen des 6. Umweltaktionsprogramms näher konkretisieren soll. Die Kommission ist der Ansicht, dass ehrgeizige Umweltziele der Wettbewerbsfähigkeit der europäischen Industrie zugute kommen werden.

Unter der Überschrift „Generationengerechtigkeit" zählt der Entwurf einer Nachhaltigkeitsstrategie für Deutschland den sparsamen Umgang mit den natürlichen Ressourcen zu den wichtigsten Zukunftsaufgaben. Ziel ist die Erhöhung der Energie- und Ressourceneffizienz - die Vision einer Effizienzsteigerung um den „Faktor 4“ oder gar den „Faktor 10“ macht danach die Richtung deutlich (Bundesregierung Deutschland 2001). Entsprechend zählt die Ressourcenintensität zu den 21 Schlüsselindikatoren für eine nachhaltige Entwicklung in Deutschland. Der reziproke Indikator - die Ressourcenproduktivität - zählt zu den 6 Leitindikatoren, die das so genannte „UmweltBarometer-Deutschland“ bilden (http://www. umweltbundesamt.de/dux/).

\section{Bedingungen einer nachhaltigen physischen Wirtschaft}

Eine Politik des nachhaltigen Ressourcenmanagements sollte sich an Kriterien für einen nachhaltigen Stoffhaushalt orientieren. Nach den bisherigen - zu einem großen Teil aus den oben vorgestellten Studien abgeleiteten - empirischen Erkenntnissen sowie aus einer (öko)systemanalytischen Perspektive können die folgenden Bedingungen einer nachhaltigen physischen Wirtschaft zusammenfassend formuliert werden (Bringezu 2002):

- Anpassung der menschlich verursachten Stoffströme an die natürlichen Tragekapazitäten: Die Entnahme von natürlichen Ressourcen aus der Umwelt und die Abgabe von Emissionen in die Umwelt kann nur so lange aufrecht erhalten bleiben, wie das Volumen und die Zusammensetzung dieses Stoffwechsels nicht die räumlichen und zeitlichen Tragekapazitäten des natürlichen Umweltsystems beeinträchtigen. Dies bezieht sich sowohl auf die lokale, regionale und globale Verfügbarkeit von Ressourcen wie auch auf die Kapazitäten des natürlichen Umweltsystems, Emissionen und Abfälle aufzunehmen. Im Hinblick auf die physischen Austauschbeziehungen zwischen Regionen impliziert diese Bedingung, dass die stofflichen $\mathrm{Zu}$ - und Abflüsse in qualitativer wie quantitativer Form ausgeglichen sein sollten. Der gegenwärtige Stoffwechsel der EU-Wirtschaft ist hiervon aufgrund des Ausmaßes nicht-erneuerbaren Ressourcenaufwandes noch weit entfernt.

- Einschränkung des physischen Wachstums der Volkswirtschaften: Mittel- bis langfristig muss das physische Wachstum industrialisierter Volkswirtschaften in ein Fließgleichgewicht von Ressourcenentnahmen und Reststoffabgaben übergehen - und zwar auf einem Niveau, welches eine langfristige Koexistenz von Mensch und Natur garantiert. Die gegenwärtige Expansion der Technosphäre - überwiegend in Form von zusätzlichen Gebäuden und Infrastrukturen - kann nicht unendlich fortgesetzt werden. Letztere Bedingung ergibt sich auch insbesondere im Hinblick auf die begrenzten Landflächen, die zur Reproduktion von Biomasse in der Land- und Forstwirtschaft benötigt werden. Der physische Kapitalbestand der Volkswirtschaften muss langfristig auf ein Niveau begrenzt werden, welches die lebenserhaltenden Funktionen der Natur nachhaltig ermöglicht. Dieses nachhaltige Niveau ist bislang noch unbekannt. Jedoch zeigt das gegenwärtige physische Wachstum bereits an, dass wir von einem Fliessgleichgewicht zwischen Materialinputs und Materialoutputs weit entfernt sind.

- Intra-generationelle Gerechtigkeit: Eine Region (Wirtschaftsraum) sollte durch seine eigene Entwicklung nicht die Entwicklungsmöglichkeiten anderer Regionen einschränken. Dies gilt nicht nur für Regionen, sondern auch für Individuen. Daher sollten die Umweltbeeinträchtigungen pro Kopf nicht ungleich verteilt sein. Dies gilt sowohl für die Nutzung natürlicher Ressourcen wie auch für die Abgabe von Emissionen und Abfällen an die Umwelt. Gegenwärtig je- 
doch besteht ein nachweisbarer Trend in der Verlagerung von Ressourcenextraktionen und ihren Umweltbelastungen in außereuropäische Regionen.

- Inter-generationelle Gerechtigkeit: Die Möglichkeiten zukünftiger Generationen sollten nicht durch die gegenwärtige Struktur und das Volumen des gesellschaftlichen Stoffwechsels beeinträchtigt werden. Sicherlich bedeutet diese Bedingung die größte Herausforderung. Sie impliziert die Heranführung der gegenwärtigen Ausprägung des gesellschaftlichen Stoffwechsels hin zu einem dynamischen und kontinuierlichen Fließgleichgewichtssystem zwischen Umwelt und Gesellschaft. „Dynamisch“ bezieht sich dabei auf den Flusscharakter (keine statische Situation), die stoffliche Zusammensetzung (die sich im Zeitverlauf ändern wird) und den (erforderlichen) technologischen Wandel. „Kontinuierlich“ wird in diesem Sinne verstanden als die Notwendigkeit, stoffliche Versorgungs- und Entsorgungssysteme zu etablieren, die langfristig aufrechterhaltbar sind.

\section{Ausblick}

Stoffbezogene Umweltpolitik in den 1970er und 1980er Jahren betonte substanz-spezifische Probleme und verfolgte somit eine „DeToxifizierung“ des gesellschaftlichen Stoffwechsels. Ab den 1990er Jahren entwickelte sich die dazu komplementäre Strategie der „Dematerialisierung“. Hierzu wurden entsprechende Methoden entwickelt, wie die wirtschaftsraumbezogene Stoffflussanalyse und davon abgeleitete Indikatoren. In Zukunft wird es nicht nur darum gehen, schädliche Stoffflüsse zu eliminieren und die Volumina menschlich verursachter Stoffströme durch immer effizientere Prozesse zu reduzieren. Es wird auch darum gehen, welche Stoffflüsse langfristig und in einer an nachhaltigen Kriterien orientierten Art und Weise aufrechterhalten werden können und sollen. Die Frage ist, auf welches Niveau und welche Zusammensetzung sich ein zukünftiger postindustrieller Stoffwechsel einpegeln muss. Erste Umrisse hierfür haben wir skizziert (siehe auch Bringezu 2002). Aber es sind noch viele Fragen zu beantworten, um zu klären, welches Volumen, welche Struktur und Zusammenset- zung ein nachhaltiger gesellschaftlicher Stoffwechsel langfristig aufweisen kann bzw. soll. Hierfür gilt es, das Wissen aus sehr verschiedenen Disziplinen zusammenzuführen. Die für die Zukunft fundamentale Frage, wie die physische Basis unserer Gesellschaft nachhaltig gesichert werden kann, wird daher auch nur in Zusammenarbeit verschiedener Forschungsinstitutionen zu beantworten sein.

\section{Anmerkungen}

1) Engl.: industrial metabolism oder societal metabolism

2) Der Begriff „Stoff“ (engl. „material“) umfasst hier die Begriffe „Material“ (engl. „bulkmaterial") und „Substanz" (engl. ,substance“)

3) Statistisches Bundesamt: Fachserie 19, Umwelt, Reihe 4, Umweltökonomische Gesamtrechnungen - Basisdaten und ausgewählte Ergebnisse 2000, Stuttgart 2001.

Statistisches Bundesamt: Fachserie 19, Umwelt, Reihe 5, Umweltökonomische Gesamtrechnungen - Material- und Energieflussrechnungen 1999, Stuttgart 2000.

\section{Literatur}

Adriaanse, A.; Bringezu, S.; Hammond, A.; Moriguchi, Y.; Rodenburg, E.; Rogich, D.; Schütz, H., 1998: Stoffströme: Die materielle Basis von Industriegesellschaften. (Wuppertal Texte) Berlin, Basel, Boston: Birkhäuser Verlag

Ayres, R.U., 1989: Industrial Metabolism. In: Ausubel, J.; Sladovich, H. (eds.): Technology and Environment, Washington D.C: National Academy

Ayres, R.U.; Simonis, U.E. (Hrsg.), 1994: Industrial Metabolism. Tokyo, New York: United Nations University Press

Baccini, P.; Brunner, P.H., 1991: Metabolism of Anthroposphere. Heidelberg, New York: Springer

Bringezu, S., 1993: Towards increasing resource productivity: How to measure the total material consumption of regional or national economies? In: Fresenius Environmental Bulletin (2), S. 437-442

Bringezu, S., 1995: Neue Ansätze in der Umweltstatistik - Ein Wuppertaler Werkstattgespräch. (Wuppertal Texte) Berlin, Basel, Boston: Birkhäuser Verlag

Bringezu, S., 1997: Umweltpolitik: Grundlagen, Strategien und Ansätze ökologisch zukunftsfähigen Wirtschaftens. München, Wien: Oldenbourg Verlag

Bringezu, S., 2000: Ressourcennutzung in Wirtschaftsräumen: Stoffstromanalysen für eine nach- 
haltige Raumentwicklung. Heidelberg, New York: Springer

Bringezu, S., 2002: Towards Sustainable Resource Management in the European Union: (Wuppertal Paper No. 121), Wuppertal: Wuppertal Institute

Bringezu, S.; Fischer-Kowalski, M.; Kleijn, R.; Palm, V. (Hrsg.), 1997: From Paradigm to Practise of Sustainability. Proceedings of the ConAccount Workshop 21 -23 January 1997 in Leiden, (Wuppertal Special 4), Wuppertal: Wuppertal Institut

Bringezu, S.; Fischer-Kowalski, M.; Kleijn, R.; Palm, V. (Hrsg.), 1998a: Analysis for Action - Support for Policy towards Sustainability by Material Flow Accounting. Proceedings of the ConAccount Conference 11 - 12 September 1997 in Wuppertal, (Wuppertal Special 6), Wuppertal: Wuppertal Institut

Bringezu, S.; Fischer-Kowalski, M.; Kleijn, R.; Palm, V. (Hrsg.), 1998b: The ConAccount Agenda: The Concerted Action on Material Flow Analysis and its Agenda for Research and Development. (Wuppertal Special 8), Wuppertal: Wuppertal Institute

Bringezu, S.; Moriguchi, Y., 2002: Material Flow Analysis. In: Ayres, R.U.; Ayres, L. (Hrsg.): Handbook of Industrial Ecology. Edward Elgar Publishers, in print

Bringezu, S.; Schütz, H., 1995: Wie mißt man die ökologische Zukunftsfähigkeit einer Volkswirtschaft? Ein Beitrag der Stoffstrombilanzierung am Beispiel der Bundesrepublik Deutschland. In: Bringezu, S. (Hrsg.): Neue Ansätze in der Umweltstatistik. Berlin, Basel, Boston: Birkhäuser Verlag, S. 26-54

Bringezu, S.; Schütz, H., 2001a: Total Material Requirement of the European Union. (EEA Technical Report No. 55), Copenhagen: European Environment Agency

Bringezu, S.; Schütz, H., 2001b: Total Material Requirement of the European Union - Technical part. (EEA Technical Report No. 56), Copenhagen: European Environment Agency

Bringezu, S.; Schütz, H., 2001c: Material use indicators for the European Union, 1980-1997, Eurostat Working Paper 2/2001/B/2, Luxemburg: Eurostat

Bundesregierung Deutschland, 2001: Perspektiven für Deutschland: Unsere Strategie für eine nachhaltige Entwicklung - Entwurf der Nationalen Nachhaltigkeitsstrategie. Berlin

Daly, H., 1991: Steady State Economics. 2nd edition, Washington: Island Press

Daly, H., 1997: Georgescu-Roegen versus Solow/ Stiglitz. In: Ecological Economics, 22 (3), S. 261-266

Enquete-Kommission ,Schutz des Menschen und der Umwelt - Bewertungskriterien und Perspektiven für umweltverträgliche Stoffkreisläufe in der Industriegesellschaft" des Deutschen Bundestag, 1994: Die
Industriegesellschaft gestalten - Perspektiven für einen nachhaltigen Umgang mit Stoff- und Materialströmen. (Bundestags-Drucksache 12/8260) Bonn

Enquete-Kommission „Schutz des Menschen und der Umwelt - Ziele und Rahmenbedingungen einer nachhaltig zukunftsverträglichen Entwicklung" des Deutschen Bundestag, 1998: Konzept Nachhaltigkeit: Vom Leitbild zur Umsetzung. (BundestagsDrucksache 13/11200) Bonn

Eurostat - Statistical Office of the European Communities (Hrsg.), 2001: Economy-wide material flow accounts and derived indicators - A methodological guide. Luxemburg: European Communities

Fischer-Kowalski, M.; Haberl, H.; Hüttler, W.; Payer, H.; Schandl, H.; Winiwarter, V.; Zangerl-Weisz, H., 1997: Gesellschaftlicher Stoffwechsel und Kolonisierung der Natur: Ein Versuch in Sozialer Ökologie. Amsterdam: Gordon Breach Fakultas

Georgescu-Roegen, N., 1971: The Entropy Law and the Economic Process. Cambridge, Mass.: Harvard University Press

Kommission der Europäischen Gemeinschaften, 2001a: 'Umwelt 2010: Unsere Zukunft liegt in unserer Hand' - Sechstes Umweltaktionsprogramm Vorschlag für einen Beschluss des europäischen Parlaments und des Rates über das Umweltaktionsprogramm 2001-2010 der Europäischen Gemeinschaft (Vorlage der Kommission). Mitteilung der Kommission KOM (2001) 31 endgültig, Brüssel, den 24.1.2001

Kommission der Europäischen Gemeinschaften, 2001b: Nachhaltige Entwicklung in Europa für eine bessere Welt: Strategie der Europäischen Union für die nachhaltige Entwicklung (Vorschlag der Kommission für den Europäischen Rat in Göteborg). Mitteilung der Kommission KOM(2001)264 endgültig, Brüssel, den 15.5.2001

Matthews, E.; Amann, C.; Bringezu, S.; FischerKowalski, M.; Hüttler, W.; Kleijn, R.; Moriguchi, Y.; Ottke, C.; Rodenburg, E.; Rogich, D.; Schandl, H.; Schütz, H.; van der Voet, E.; Weisz, H., 2000: The Weight of Nations - Material Outflows of Industrial Economies. Washington: Word Resources Institute

Radermacher, W.; Stahmer, C., 1994: Vom Umwelt-Satellitensystem zur Umweltökonomischen Gesamtrechnung - Umweltbezogene Gesamtrechnungen in Deutschland, Teil 1. In: Zeitschrift für angewandte Umweltforschung, H. 4/1994, S. 531 ff.

Radermacher, W.; Stahmer, C., 1995: Vom UmweltSatellitensystem zur Umweltökonomischen Gesamtrechnung - Umweltbezogene Gesamtrechnungen in Deutschland, Teil 1. In: Zeitschrift für angewandte Umweltforschung, Teil 2: H. 1/1995, S. 99 ff.

Radermacher, W.; Stahmer, C., 1997: German Material and Energy Information System. In: Bartelmus, 
P.; Uno, K. (Hrsg.): Environmental Accounting in Theory and Practice. Dordrecht: Kluwer

Schmidt-Bleek, F.; Bringezu, S.; Hinterberger, F.; Liedtke, C.; Stiller, H.; Spangenberg, J.; Welfens, M.J., 1998: MAIA: Einführung in die Material-Intensitäts-Analyse nach dem MIPS-Konzept. (Wuppertal Texte) Berlin, Basel, Boston: Birkhäuser Verlag

Statistisches Bundesamt (Hrsg.), 1997: AGME Arbeitsgemeinschaft Material- und Energieflussrechnung. (Broschüre); Wiesbaden: Statistisches Bundesamt

Strassert, G., 1993: Towards an Ecological Accounting of the Provision-TransformationRestitution Cycle. In: Dragan, J.C.; Seifert, E.K., Demetrescu, M.C. (Hrsg.): Entropy and Bioeconomics, Milano: Nagard, S. 507-515

United Nations, 1993: Integrated Environmental and Economic Accounting - Interim Version. Handbook of National Accounting, Series F, No. 61, New York: United Nations

United Nations, 2000: Integrated Environmental and Economic Accounting - An Operational Manual. New York: United Nations

\section{Kontakt}

Dr. Stefan Bringezu

Wuppertal Institut für Klima, Umwelt, Energie

Postfach 100480, 42004 Wuppertal

Tel.: +49 (0) 202 / 2492131

E-Mail: Stefan.Bringezu@wupperinst.org

\section{Ein Beitrag zur Bewertung des Stoffhaushaltes von Metallen}

\author{
von Dipl. Ing. Dr. Helmut Rechberger, Eidge- \\ nössische Technische Hochschule Zürich
}

\begin{abstract}
Schwermetalle stellen einen wesentlichen Bestandteil des Güter- und Stoffumsatzes entwickelter Volkswirtschaften dar. Dabei werden sie einerseits in Veredelungsprozessen konzentriert, andererseits in Konsumprozessen verdünnt und emittiert. Es wird eine Methode vorgestellt, die es erlaubt, diese Vorgänge zu quantifizieren. Die neue Messgröße kann als ein Bewertungsindikator auf dem Weg zu einer nachhaltigeren Metallbewirtschaftung herangezogen werden.
\end{abstract}

\section{Einleitung}

Moderne Volkswirtschaften setzen steigende Mengen an materiellen Gütern pro Zeiteinheit um. Dieser Anstieg ist durch Statistiken gut dokumentiert und zeigt für die meisten Güter eine ähnliche Verbrauchsentwicklung, nämlich, dass ca. 80-90\% des Gesamtverbrauches in der zweiten Hälfte des 20. Jahrhunderts stattgefunden haben und der Trend ein weiter steigender ist. Metalle gehören dabei massemäßig nicht zu den dominierenden Gütern. Sie stellen einen Anteil $<10 \%$ an den konsumierten anorganischen Gütern dar (Baccini und Bader 1996). Sie sind jedoch auf Grund ihrer chemischphysikalischen Eigenschaften wichtig für die Produktion von Gütern und in der Regel relevant für den Umweltschutz. Das bedeutet, Metalle sind wesentliche Ressourcen und potenzielle Schadstoffe und bedürfen daher einer kontrollierten Bewirtschaftung und Steuerung innerhalb der Volkswirtschaft. Eine Grundlage dazu leisten Stoffbilanzen ${ }^{1}$, die den Weg eines Metalls vom ursprünglichen Erz über die Verarbeitung, Nutzung, Wiedergewinnung (Recycling) und kontrollierten Entsorgung in Deponien bis hin zur diffusen Emission und Ablagerung in der Umwelt zeichnen. Sobald diese Zusammenhänge bekannt und geeignete legistische, ökonomische oder technische Stellgrößen identifiziert sind, kann der Stoffhaushalt des Systems (hier der Volkswirtschaft) verbessert werden. Hierbei spricht man meist davon, das System nachhaltiger zu gestalten. Nachhaltigkeit als Paradigma, 